\title{
FBXO17 promotes cell proliferation through activation of Akt in lung adenocarcinoma cells
}

Tomeka L. Suber ${ }^{1,4}$, Ina Nikolli $i^{1,4}$, Michael E. O'Brien ${ }^{1,4}$, James Londino ${ }^{1,4}$, Jing Zhao ${ }^{1,4}$, Kong Chen ${ }^{1,4}$, Rama K. Mallampalli, ${ }^{1,2,3,4^{*}+}$ (D) and Yutong Zhao ${ }^{1,4^{*}+}$

\begin{abstract}
Background: The ubiquitin-proteasome pathway, mediated in part, by ubiquitin E3 ligases, is critical in regulating cellular processes such as cell proliferation, apoptosis, and migration. FBXO17 was recently identified as an F-box protein that targets glycogen synthase kinase-3 $\beta$ to the E3 ubiquitin ligase protein complex for polyubiquitination and proteasomal degradation. Here, we identified that in several lung adenocarcinoma cell lines, FBXO17 cellular protein was detected at relatively high levels, as was expression in a subset of lung cancers. Hence, we investigated the effects of FBXO17 on cell proliferation.

Methods: Single cell RNA sequencing analysis was performed on a resection of a non-small cell lung carcinoma tumor to examine FBXO17 expression. Multiple lung cancer cell lines were immunoblotted, and The Cancer Genome Atlas was analyzed to determine if FBXO17 expression was amplified in a subset of lung cancers. A549 cells were transfected with empty vector or FBXO17-V5 plasmid and immunoblotted for Akt pathway mediators including PDK1, ERK1/2, ribosomal protein S6, and CREB. Cell proliferation and viability were analyzed by trypan blue exclusion, BrdU incorporation and an MTS-based fluorometric assay. Studies were also performed after transfecting with sifbxo 17. Samples were used in an RNA microarray analysis to evaluate pathways affected by reduced FBXO17 gene expression.

Results: We observed that overexpression of FBXO17 increased A549 cell proliferation coupled with Akt activation. Ectopically expressed FBXO17 also increased ERK1/2 kinase activation and increased phosphorylation of RPS6, a downstream target of mTOR. We also observed an increased number of cells in S-phase and increased metabolic activity of lung epithelial cells expressing FBXO17. FBXO17 knockdown reduced Akt Ser 473 phosphorylation approaching statistical significance with no effect on Thr 308. However, ERK1/2 phosphorylation, cellular metabolic activity, and overall cell numbers were reduced. When we analyzed RNA profiles of A549 cells with reduced FBXO17 expression, we observed downregulation of several genes associated with cell proliferation and metabolism.

Conclusions: These data support a role for FBXO17 abundance, when left unchecked, in regulating cell proliferation and survival through modulation of Akt and ERK kinase activation. The data raise a potential role for the F-box subunit in modulating tumorigenesis.
\end{abstract}

Keywords: Akt, FBXO17, Proliferation, Lung cancer

\footnotetext{
*Correspondence: mallampallirk@upmc.edu; zhaoy3@upmc.edu

${ }^{+}$Rama K. Mallampalli and Yutong Zhao contributed equally to this work.

'Department of Medicine, the Acute Lung Injury Center of Excellence,

University of Pittsburgh, Pittsburgh, PA 15213, USA

Full list of author information is available at the end of the article
}

(c) The Author(s). 2018 Open Access This article is distributed under the terms of the Creative Commons Attribution 4.0 International License (http://creativecommons.org/licenses/by/4.0/), which permits unrestricted use, distribution, and reproduction in any medium, provided you give appropriate credit to the original author(s) and the source, provide a link to the Creative Commons license, and indicate if changes were made. The Creative Commons Public Domain Dedication waiver (http://creativecommons.org/publicdomain/zero/1.0/) applies to the data made available in this article, unless otherwise stated. 


\section{Background}

The phosphatidylinositol-4,5-bisphosphate 3-kinase (PI3K)Akt- mammalian target of rapamycin (mTOR) pathway drives many cellular functions including cell survival, proliferation, and migration. Activation of receptor tyrosine kinases (RTKs) including EGFR, Her2/Neu, and VEGF activates and recruits PI3K to the plasma membrane [1]. Subsequent phosphorylation of phosphatidylinositol-4,5-bis phosphate $\left(\mathrm{PIP}_{2}\right)$ generates phosphatidylinositol-3,4,5-trisphosphate $\left(\mathrm{PIP}_{3}\right)$ as a second messenger to recruit and activate Akt through phosphorylation by PDK1. Akt then phosphorylates downstream targets such as Bad, GSK3 $\beta$, and FOXO transcription factors to inhibit apoptosis and promote cell survival, migration, and proliferation $[2,3]$. Akt activation occurs on serine 473, the obligatory catalytic site, and threonine 308 , a tightly regulated site that is required for maximal Akt activation. Phosphatase and tensin homolog (PTEN) is well-characterized as the critical negative regulator of Akt activity through dephosphorylation of $\mathrm{PIP}_{3}$ to $\mathrm{PIP}_{2}$ [4]. A critical substrate of Akt is mTOR, a serine-threonine kinase that forms part of two complexes mTORC1 and mTORC2. While the primary targets of mTORC1 include p70S6 Kinase 1 (S6K1), RPS6, and eIF4E Binding Protein (4EBP) to promote protein translation, mTORC2 facilitates cell growth through phosphorylation of downstream targets such as several members of the AGC (PKA/PKG/PKC) family of kinases in addition to Akt $[5,6]$.

Mutations, amplifications, and hyperactivity of the PI3KAkt-mTOR pathway can lead to aberrant cell growth and tumorigenesis [7]. Activating mutations in PIK3CA encoding PI3K occur in a large number of lung cancers $[8,9]$. Mutations in PTEN are among the highest frequency mutations in all cancers [10-12]. A large number of mTOR mutations have been identified in several malignancies, some of which confer constitutive activation to the kinase [13]. A majority of lung cancers have high levels of mTOR pathway activation, and phosphorylation of S6K is associated with metastasis and poor survival in adenocarcinoma [14]. Developing therapies with more specific targeting of the mTOR pathway based on molecular profiling of tumors is an intense area of research. In non-small cell lung cancers (NSCLC), mutations in NF1, MET, and ERBB2 account for up to $13 \%$ of tumors analyzed by molecular profiling, and elevation in MAPK and PI3K activity was observed in a large proportion of cases [15].

The cellular concentrations of key effectors that drive malignant phenotypes within cellular signaling pathways such as the PI3K/Akt/mTOR signaling cascade are partly controlled at the level of protein stability [16-18]. The ubiquitin-proteasome pathway is the primary mechanism for degradation of cellular proteins in eukaryotic cells $[11,19]$. Regulation of protein stability is critical for cellular homeostasis, and disruption can lead to aberrant cell proliferation. The final step in targeting proteins for proteasomal degradation is transfer of polyubiquitin chains to the targeted substrates by an E3 ubiquitin ligase. The Skp-Cullin-F-box (SCF) family is the largest family of E3 ubiquitin ligases, comprised of multiple subunits that execute ubiquitination of targets through a substrate recognition module, termed an F-box protein. There are $\sim 70$ F-box proteins, many of which have not been characterized [20]. Proteins undergo post-translational modifications, usually phosphorylation, to generate a "degron" that is recognized by the E3 ubiquitin ligase complex [21, 22] . Dysregulation of several F-box proteins have been linked to cancer. For example, Fbxw7 targets mTOR, c-Myc, c-Jun, cyclin E, and several other proteins implicated in oncogenesis, thus functioning as a tumor suppressor [23]. Mutations in $F B X W 7$ are highly represented in bile duct cancers and T-cell acute leukemia, and a large proportion are located in the domain required for substrate recognition [24]. Bcl-6, a proto-oncogene overexpressed in diffuse large B-cell lymphoma (DLBCL), is targeted by FBXO11 for polyubiquitination and degradation [25]. In a number of DLBCL lines FBXO11 was found to be mutated or deleted, and restoration of FBXO11 expression in DLBCL-derived tumor cells in immunodeficient mice induced apoptosis and suppressed tumor growth.

A poorly studied F-box protein, FBXO17, was recently found to be robustly expressed in murine and human lung alveolar epithelial cells [26]. We previously characterized FBXO17 as a negative regulator of glycogen synthase kinase-3 $\beta$ (GSK3 $\beta$ ) through polyubiquitination and targeting of the kinase to the proteasome for degradation [26]. Because Akt phosphorylates and negatively regulates GSK3 $\beta$, a potentially important association that might impact cell growth and survival, we tested the hypothesis that FBXO17, highly expressed in lung carcinoma, regulates cell proliferation through Akt activation. We found that FBXO17 promotes cell proliferation with associated PI3K/Akt/mTOR and ERK pathway activation.

\section{Methods \\ Antibodies and reagents}

FBXO17 antibody was obtained from Origene (cat\# TA331636, Rockville, MD, dilution 1:1000). HRP-conjugated anti-mouse $\operatorname{IgG}$ and anti-rabbit IgG were obtained from BioRad (Hercules, CA, dilution 1:2000). Antibodies against Akt (cat\# 2920S, dilution 1:1000), p-Akt-Ser473 (cat\# 4060S, dilution 1:1000), p-Akt-Thr308 (cat\# 13038S, dilution 1:1000), GSK3 $\beta$ (cat\# \#9315, dilution 1:1000), p- GSK3 $\beta$ (Ser9) (cat\# 9336, dilution 1:1000), CREB (cat\# \#9197, dilution 1:1000), p-PDK1 (cat\# 3438, dilution 1:1000), ERK1/2 (cat\# 9101S, dilution 1:1000), p-ERK1/2 (cat\# 4696S, dilution 1:1000) p-mTOR (cat\# $5536 \mathrm{~T}$, dilution 1:1000), and mTOR (cat\# $2983 \mathrm{~T}$, dilution 1:1000) were obtained from Cell Signaling (Beverly, MA). PDK1 (cat\# ab52893, dilution 
1:1000) and p-CREB (cat\# ab32096, dilution 1:1000) antibodies were purchased from Abcam (cat\# ab52893, Cambridge, MA). RPS6 antibody (cat\# sc-74459, dilution 1:1000) was purchased from Santa Cruz. $\beta$ actin antibody was obtained from Sigma (St. Louis, MO, USA, dilution 1:30000). Mouse anti-V5 antibody (ref\# 46-0705, dilution 1:5000) and midiprep kits were purchased from Invitrogen (Grand Island, NY). BrdU flow cytometry assay was purchased from BD Biosciences (San Jose, CA). Lipofectamine 3000 was purchased from Thermo Fisher (Waltham, MA) and Gene Mute was purchased from SignaGen (Rockville, MD). Trypan blue stain (0.4\%) was purchased from GIBCO (Life Technologies, Grand Island, NY).

\section{Cell culture and transfection}

Human A549 cells were obtained from ATCC (Manassas, VA, USA). RPMI medium was purchased from GIBCO (Life Technologies, Grand Island, NY). Cells were supplemented with $10 \%$ fetal bovine serum (FBS) from Gemini (Sacramento, CA, USA). Cloning of FBXO17 was described previously [26]. Cells were seeded at a density of $5 \times 10^{5}$ in a 6-well plate and transfected with $2 \mu \mathrm{g}$ FBXO17-V5 plasmid or empty vector with Lipofectamine 3000 according to the manufacturer's protocol. For gene silencing, scrambled and silencing siRNAs were purchased from Integrated DNA Technologies (Coralville, IA). Scrambled control siRNA (cat\# 51-01-19-09) and two siRNAs targeting FBXO17 (design ID\# hs.Ri.FBXO17.13.1 and hs.Ri.FBXO17.13.2) were used in dual transfections for knockdown for in vitro and immunoblotting experiments. Gene Mute was used for transfection according to manufacturer's protocol. Lysates were prepared 72 and $96 \mathrm{~h}$ post-transfection.

\section{Immunoblotting}

Cell lysates in $150 \mu \mathrm{l}$ of lysis buffer $(20 \mathrm{mM}$ Tris- $\mathrm{HCl}$ (pH 7.4), 150 mM NaCl, 2 mM EGTA, 5 mM $\beta$-glycerophosphate, $1 \mathrm{mM} \mathrm{MgCl2}$, 1\% Triton X-100, $1 \mathrm{mM}$ sodium orthovanadate, $10 \mu \mathrm{g} / \mathrm{ml}$ protease inhibitors, $1 \mu \mathrm{g} / \mathrm{ml}$ aprotinin, $1 \mu \mathrm{g} / \mathrm{ml}$ leupeptin, and $1 \mu \mathrm{g} / \mathrm{ml}$ pepstatin) were sonicated on ice for $12 \mathrm{~s}$ and centrifuged at $10000 \times \mathrm{g}$ for $10 \mathrm{~min}$ at $4{ }^{\circ} \mathrm{C}$ in a microcentrifuge. After overexpression, lysates were prepared at $48 \mathrm{~h}$ post-transfection. For knockdown, lysates were prepared $72 \mathrm{~h}$ post-transfection. For immunoblotting, equal amounts of supernatant (15 $\mu \mathrm{g}$ protein) were subjected to $10 \%$ SDS-PAGE gels, transferred to nitrocellulose membranes, blocked with $5 \%$ $(w / v)$ bovine serum albumin in TBST $(25 \mathrm{mM}$ Tris- $\mathrm{HCl}$, $\mathrm{pH} 7.4,137 \mathrm{mM} \mathrm{NaCl}$, and $0.1 \%$ Tween 20) for $1 \mathrm{~h}$, and incubated with primary antibodies in $5 \%(\mathrm{w} / \mathrm{v})$ BSA in TBST for 1-2 h. The membranes were washed at least three times with TBST at 10-min intervals followed by a $1 \mathrm{~h}$ incubation with mouse, rabbit, or goat horseradish peroxidase-conjugated secondary antibody (1:2000). The membranes were developed with an enhanced chemiluminescence detection system according to manufacturer's instructions.

\section{Cell cycle and viability assays}

For cell counting, $5 \times 10^{5}$ cells were seeded per well in a 6-well plate and transfected with FBXO17-V5 or empty plasmid. After $48 \mathrm{~h}$, cells were trypsinized and diluted 1:1 in trypan blue in a 96-well plate for cell counting. Cell viability was determined by trypan exclusion and 20 fields were counted per condition using an automated cell counter (TC20, BioRad). For FBXO17 silencing, scrambled or siFBXO17 siRNAs were transfected after seeding $2 \times 10^{5}$ cells/well. After $96 \mathrm{~h}$, cells were counted as described with viability determined by trypan blue exclusion. For overexpression, A549 cells were plated at $5 \times 10^{4}$ cells per well in triplicate per condition in a 96-well plate $24 \mathrm{~h}$ after transfection of FBXO17-V5 plasmid in $100 \mathrm{uL}$. At 24, 48, and $72 \mathrm{~h}$ after seeding, the MTS ([3-(4,5-dimethylthiazol-2-yl)-5-(3-carboxymethoxyphenyl)-2-(4-sulfophenyl)-2H-tetrazolium) assay with conversion to a soluble formazan product was performed according to manufacturer's protocols (Promega CellTiter $96^{\circ}$ AQueous One Solution Cell Proliferation Assay). After adding $20 \mathrm{uL}$ of reagent, the plate was read at $490 \mathrm{~nm}$ for absorbance per protocol. For knockdown experiments, $5 \times 10^{4}$ cells/well were seeded $48 \mathrm{~h}$ post transfection. The assays were performed at 24, 48, and $72 \mathrm{~h}$ after seeding. For cell cycle assays, A549 cells were transfected with empty vector or FBXO17-V5 plasmid. After $24 \mathrm{~h}$, cells were cultured in serum-free medium overnight for $16 \mathrm{~h}$. Cells were then pulsed with BrdU for $45 \mathrm{~min}$ and returned to culture in 10\% serum for $6 \mathrm{~h}$. Cells were fixed, permeabilized, and treated with DNAase prior to staining with anti-BrdU-APC antibody according to the manufacturer's protocol. 7-AAD was added and cells were analyzed by flow cytometry. Data analysis was performed using FlowJo Software (version 10.4.1). Similar analysis was performed for knockdown at $72 \mathrm{~h}$ post-transfection.

\section{Single cell RNA sequencing}

To determine the cell specificity of FBXO17 expression, we analyzed single-cell gene expression data from a fresh surgical resection of a non-small cell lung carcinoma tumor generated by $10 \mathrm{x}$ Genomics, Inc. (https://sup port.10xgenomics.com/single-cell-vdj/datasets/2.1.0/vdj_ v1_nsclc_5gex). Two-dimensional T-distributed stochastic neighbor embedding (t-SNE) plots were generated based on global gene expression relationships among 7,802 total cells. FBXO17 and three marker genes, including PTPRC (leukocytes), SCGB1A1 (club cells), and 
EPCAM (lung epithelial cells) were displayed with level of expression indicated by intensity of red.

\section{RNA microarray analysis and gene enrichment analysis}

A549 cells were transfected with control scrambled siRNAs or siFBXO17 (Integrated DNA Technologies). After $72 \mathrm{~h}$ cells were harvested and RNA isolated using the Invitrogen RNEasy Plus kit (3 independent control and 3 siFBXO17 samples). After confirming knockdown of FBXO17 expression by quantitative real time PCR, RNA was analyzed using the Clariom S Assay (Affymetrix and Thermo Fisher Scientific, cat\# 902926). Preparation of cDNA was performed using the WT Plus reagent kit (Thermo Fisher) and according to manufacturer instructions. Reverse transcription was performed using $100 \mathrm{ng}$ total RNA and dNTP-T7 random primers. Second strand synthesis and in vitro transcription created amplified cRNA. cDNA from a second reverse transcription reaction was fragmented and end labeled with biotin for hybridization to the Clariom $S$ human array. Following overnight (16 h) hybridization at $45{ }^{\circ} \mathrm{C}$ with rotational mixing at $60 \mathrm{rpm}$, arrays were processed on a GeneChip 450 Fluidics Station using manufacturer specified protocols. To remove unbound sample, arrays were first washed with non-stringent wash buffer A. The GeneChips ${ }^{\circ}$ were then stained for 10 min in Stain Cocktail 1. Buffer A was again used to wash off excess stain. Signal amplification was achieved by 10 min incubation with Stain Cocktail 2 followed by a second 10 min incubation with Stain Cocktail 1. The chip was washed with high stringency Buffer B and filled with Array Holding Buffer before being removed from the fluidics station and scanned using the GeneArray 3000 scanner. First level image analysis was performed using Affymetrix Expression Console. Normalization was via robust multiarray average (RMA).

\section{Gene enrichment analysis}

For pathways analysis, average log 2-fold change expression was determined in each of three technical replicates and an average fold change compared to Fbxo17 knockdown was calculated. Differentially expressed genes (DEGs) used in pathway and Gene Ontology (GO) analysis were determined using Transcriptome Analysis Console version 4.0.1 (Applied Biosystems, Foster City, CA). Filters were used to select genes with absolute fold change $(\mathrm{FC})<-1.5$ and $>1.5$ and a $P$-value $\leq 0.05$. Gene Ontology (GO) enrichment analysis was performed using ToppGene (http://toppgene.cchmc.org/) [27]. Correction for multiple comparisons was performed using the Benjamini-Hochberg procedure and significance determined using a false discovery rate (FDR) $P$-value cut-off $<0.05$. Activation or inhibition of gene pathways was evaluated with Ingenuity
Pathway Analysis (IPA, Qiagen), which compares results to experimentally determined gene expression changes reported in the literature.

\section{Statistical analysis}

Descriptive statistics were reported with mean \pm standard deviation (SD) or standard error (SE) indicated. Our sample sizes of each experimental group were all less than 10 that limited ability to study a normal sample distribution. Thus, we employed appropriate non-parametric methods such as a Mann-Whitney U test and a Kruskal-Wallis equalityof-populations rank test to compare multiple groups within and between experiments. Also, a Wilcoxon-type test for trend was used where appropriate to check trends in data significance. Using these methods provided conservative analysis to determine statistical significance. Densitometry was performed using Image J software Version 2.0.0-rc-67/ $1.52 \mathrm{~d}$ [28]. All analyses were performed using one-way ANOVA or paired t-tests using GraphPad Prism version 7.0 for Windows and Mac OS, GraphPad Software, La Jolla California USA, www.graphpad.com.

\section{Results}

FBXO17 is highly expressed in some lung cancer cell lines We first examined if FBXO17 was overexpressed or altered in lung cancer cell lines (Fig. 1a). Relative to several cell lines examined, COR-L23, COLO-699, and A549 expression of FBXO17 protein was detected most abundantly. A549 cells express 40\% more FBXO17 protein relative to normal lung tissue (Fig. 1b). We also interrogated the cBio Cancer Genomics Portal and found that FBXO17 expression was increased in 5\% of all lung cancer samples in the database $(n=1144)[15,29,30]$. Amplification of gene expression was disproportionately high in squamous cell lung cancers at $7.6 \%$ of cases compared to $2.9 \%$ of non-small cell lung cancer (NSCLC) cases (Fig. 1c). Single cell RNA-sequencing data was obtained from resected squamous cell carcinoma tumor from $10 x$ Genomics, Inc. (Fig. 1d). As shown in the t-distributed stochastic neighbor embedding (tSNE) plot, FBXO17 co-localizes with the epithelial cell marker EPCAM and is highly expressed in epithelial cells while there was no expression in leukocytes (PTPRC expression) or club cells (SGCB1A1). These data suggest that FBXO17 may be dysregulated in a subset of bronchogenic carcinomas.

\section{FBXO17 increases activation of the PI3K-Akt-mTOR signaling pathway in A549 cells}

Given our prior studies supporting a role for FBXO17 in targeting GSK3 $\beta$ for proteasomal degradation in lung epithelial cells, we examined whether FBXO17 modulates the activity of Akt, a key negative regulator of GSK3 3 . When FBXO17 was ectopically expressed in A549 cells, phosphorylation of threonine 308 (Thr 308), and to a lesser 


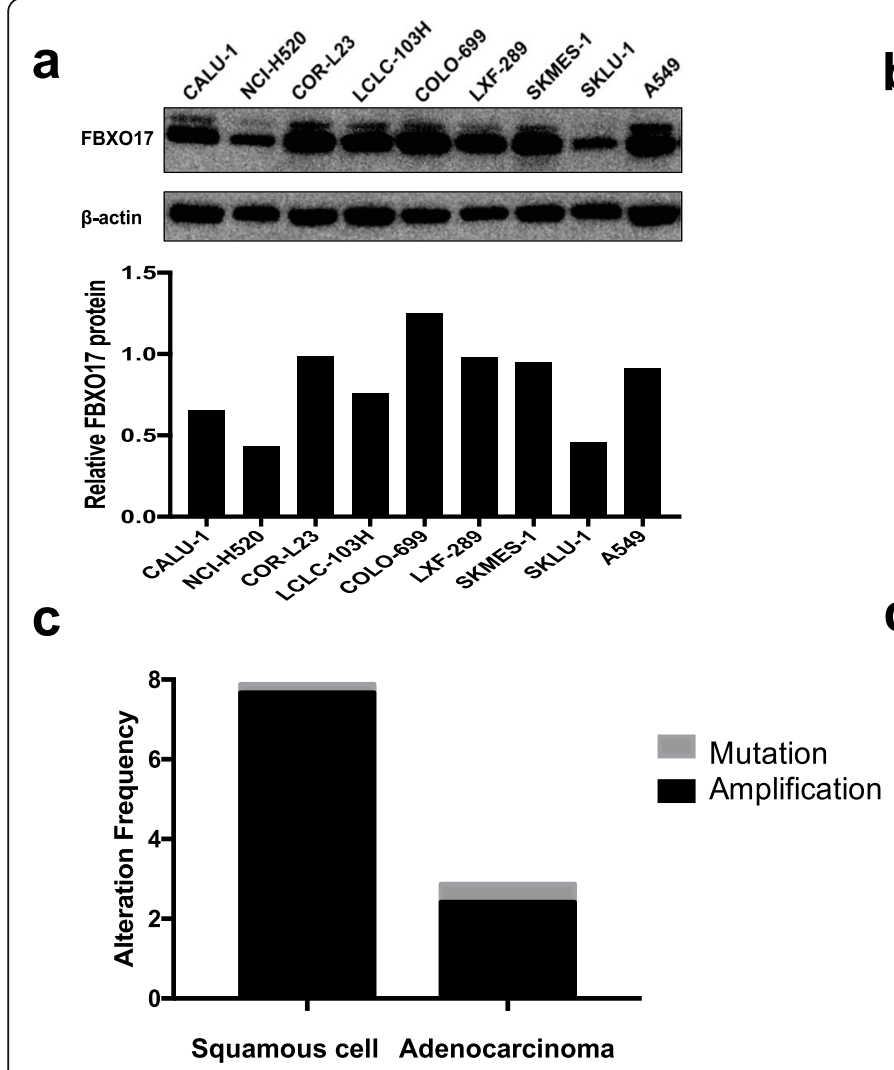

b

IB: FBX017

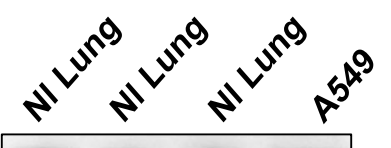

IB: $\beta$-actin

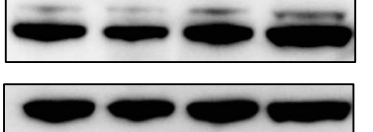

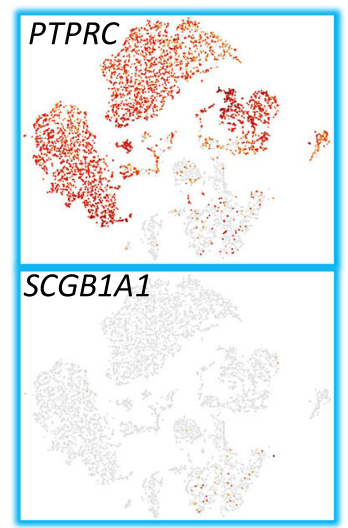

Fig. 1 FBXO17 is highly expressed in a subset of lung cancer cell lines and primary lung bronchogenic tumors. a Cell lysates of lung cancer cell lines were immunoblotted for FBXO17 protein expression with $\beta$-actin as a loading control. b Normal lung tissue and A549 lysates were immunoblotted for FBXO17 protein with $\beta$-actin as a loading control. c FBXO17 gene expression amplification was observed in a subset of lung squamous cell carcinoma and adenocarcinoma cases. The results presented here are in whole or part based upon data generated from the Cancer Genome Atlas Network: http://cancergenome.nih.gov/ ( $n=1144$ samples). d RNA-sequencing of resected non-small cell lung cancer tumor is displayed in a t-distributed stochastic neighbor embedding (tSNE) plot showing RNA expression of cell-specific markers including epithelial adhesion molecule (EPCAM, epithelial cells), protein tyrosine phosphatase receptor type C (PTPRC, leukocytes), and secretoglobin family $1 \mathrm{~A}$ member 1 (SCGB1A1, club cells)

degree serine 473 (Ser 473) were increased relative to total Akt levels (Fig. 2a). Inhibitory Ser 9 phosphorylation of GSK3 $\beta$ was unchanged with modest reduction in total protein levels, consistent with prior published data [26]. In addition, phosphorylation of 3-phosphoinositide dependent protein kinase 1 (PDK1), extracellular signal-regulated kinase-1/2 (ERK1/2), p-CREB, and RPS6 were increased after ectopic FBXO17 expression (Fig. 2a, b). With knockdown, there was a trend for a reduction of p-Akt (Ser473) phosphorylation while p-Akt (Thr308) was not significantly decreased. (Fig. 2c). We observed significant reductions in p-ERK1/2 with FBXO17 knockdown while PDK1 phosphorylation also decreased but did not reach significance after densitometric analysis of bands visualized on the immunoblots. The data show that robust expression of FBXO17 is associated with increased Akt, PDK1 and ERK1/2 activation, with more modest effects upon FBXO17 cellular depletion.

\section{FBX017 increases A549 cell proliferation}

To determine if FBXO17 influenced cell survival and growth, FBXO17-V5 plasmid was overexpressed in A549 cells. At $48 \mathrm{~h}$ post-transfection cell numbers significantly increased with FBXO17 overexpression but not with empty vector (Fig. 3a). In a metabolic MTS colorimetric assay, cell viability was also modestly enhanced at 24,48 , and $72 \mathrm{~h}$ post transfection (Fig. 3b). After $16 \mathrm{~h}$ of serum deprivation, cells were pulsed with BrdU and stimulated with $10 \%$ serum prior to staining and flow cytometric analysis. A higher fraction of cells was found to be in $\mathrm{S}$ phase with $F B X O 17$ overexpression compared to empty vector (Fig. 3c, d). When FBXO17 expression was silenced, cell proliferation was significantly reduced (Fig. 4a, b). These results support a role for a high level of FBXO17 protein mass in modulating cell proliferative behavior, and depletion of $F B X O 17$ gene expression substantially impairs cell growth and survival. 


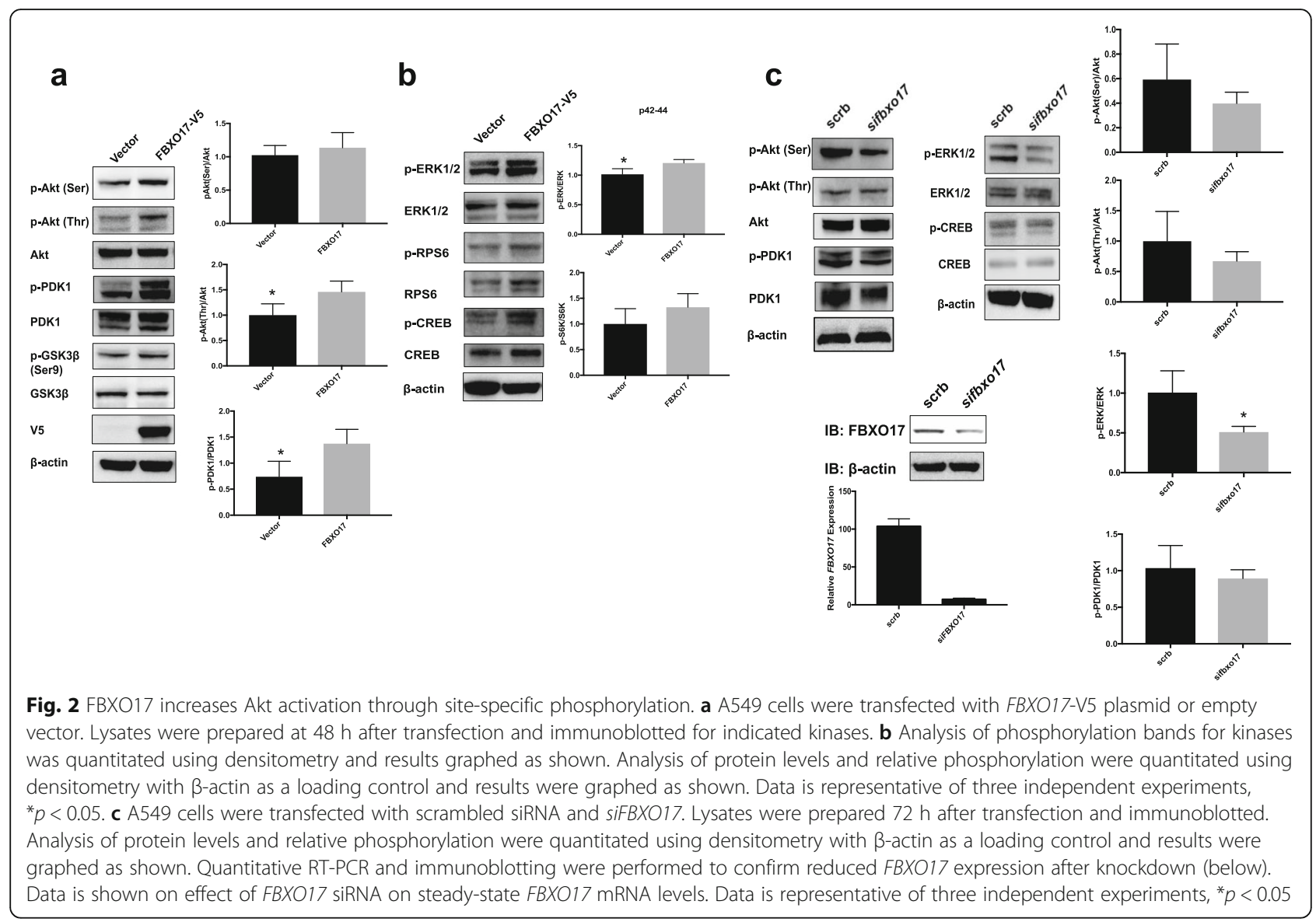

\section{Reduced expression of FBXO17 enriches for squamous and adenocarcinoma-associated genes}

When we examined pathways affected by knockdown of FBXO17 using RNA microarray analysis, we identified enrichment of genes affected which included endopeptidase proteins and pathways, hypoxia-inducible factor 1-alpha (HIF1 $\alpha)$ pathway genes, and both squamous cell cancer and adenocarcinoma-associated genes (Fig. 4c-f). Gene expression analysis revealed 212 genes that were differentially expressed in A549 cells that underwent FBXO17 knockdown (100 downregulated and 112 upregulated) (Fig. 4c). Pathway analysis revealed decreased expression of nine genes involved in cell proliferation and metabolism of tumor cell lines, indicating a role for upstream inhibition of the transcriptional regulator ATF4 and upstream activation of let-7 in our model of FBXO17 knockdown (Fig. 4d). We confirmed CKS1B reduction by immunoblotting (Fig. 4e). Gene enrichment analysis of these differentially expressed genes revealed that regulation of endopeptidase and peptidase activity (FDR $p$-value 5.84 e-03) were the most significant biological processes involved in the experimental model of FBXO17 knockdown. Enzymatic regulators of extracellular matrix remodeling were the most significant pathway affected (FDR
$P$-value 2.87 e-04). Adenocarcinoma was the disease category most significantly associated with DEGs in our model (FDR P-value 8.97 e-03), (Fig. 4f). Additional genes and pathways that are differentially expressed are included in Additional files 1 and 2. In summary, several genes shown to be differentially expressed when FBXO17 is depleted are implicated in metabolic and cell proliferative functions that may provide further insight into the role of this poorly studied F-box protein in cancer biology.

\section{Discussion}

The role of FBXO17 in the regulation of cell growth and survival to date is unknown. FBXO17 mediates degradation of GSK3 $\beta$, a purported inhibitor of cell proliferative activity, that in turn is inhibited by Akt [31]. These data together with findings that Akt participates in the pathobiology of tumorigenesis led us to investigate if FBXO17 is differentially expressed in lung carcinoma. Indeed, FBXO17 was detected at high levels in many lung carcinoma cell lines and its transcript is highly expressed in squamous cell lung cancer. When overexpressed in A549 cells, FBXO17 increased cellular proliferative activity by $\sim 25 \%$ coupled with increased survival of cells. We also demonstrate here that Akt phosphorylation 


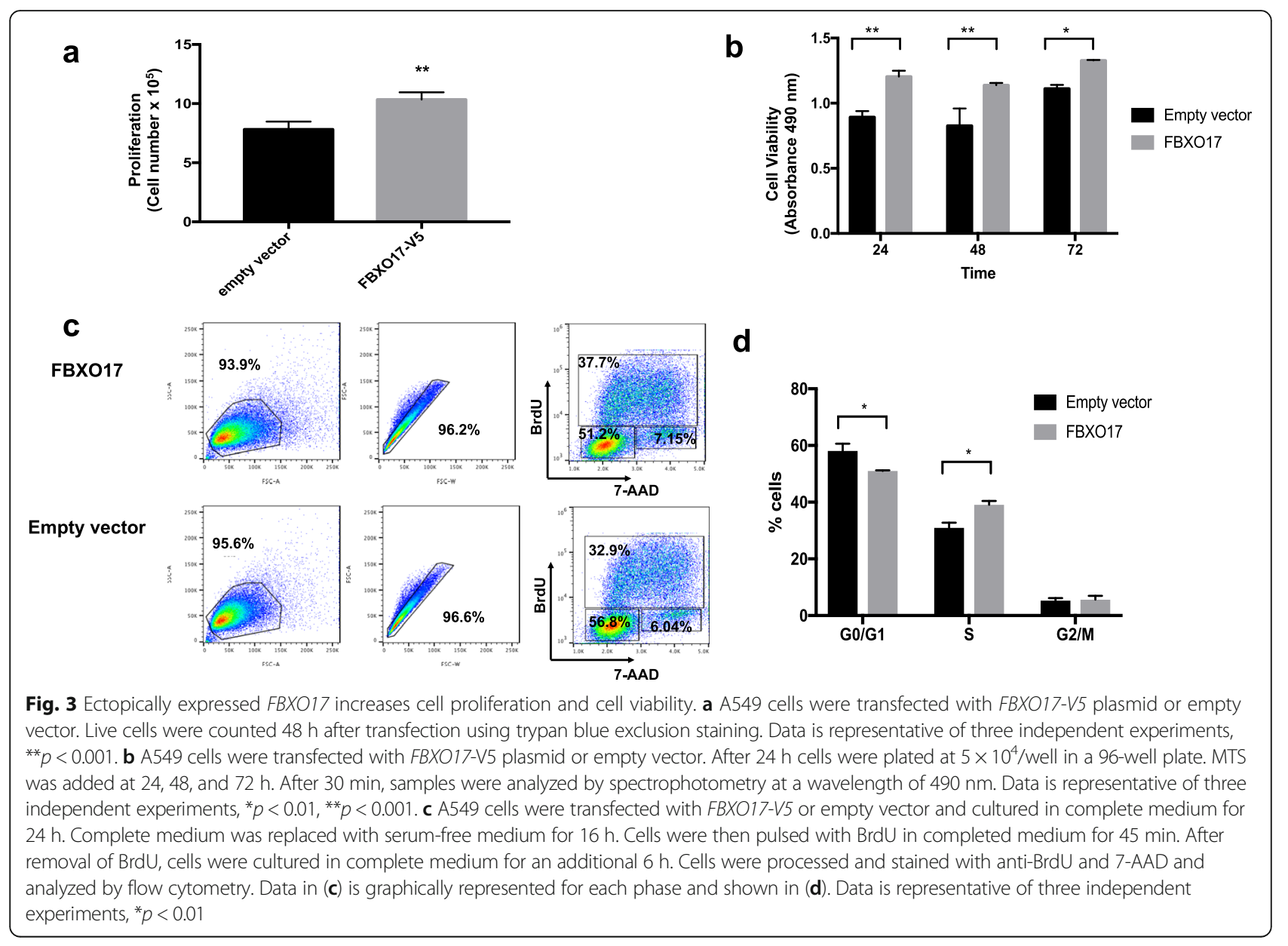

on Thr 308, and to a lesser extent Ser 473, is enhanced by ectopic FBXO17 expression. Interestingly, Ser 473 phosphorylation was reduced more than Thr 308 with FBXO17 knockdown. Our studies support a correlation between higher levels of FBXO17 and Thr 308 activity. A number of genes implicated in key pathways and biological processes that are linked to tumorigenesis were modulated after $F B X O 17$ silencing in cells. These results suggest that FBXO17 is sufficient to elicit pro-survival effects in some cell lines. Depletion of FBXO17 gene expression substantially decreases replicative behavior of these cells.

Proliferation of A549 cells was clearly enhanced by ectopic FBXO17 expression. Akt activation by PDK1 occurs in a two-step process. Serine 473 phosphorylation is obligatory for activation; however, maximal activation requires subsequent phosphorylation of threonine 308 [2, 6, 32]. Phosphorylation of both sites is tightly regulated and influences downstream signaling and substrate selection. It has been proposed that the balance of Ser 473 and Thr 308 phosphorylation is integrated to regulate downstream signals [2]. Akt activity is fine-tuned by phosphorylation on additional sites by other upstream kinases [33]. In our overexpression studies, ectopically expressed FBXO17 seemed to clearly upregulate Thr 308 phosphorylation to a greater extent than serine 473. The converse was true with knockdown where Ser 473 phosphorylation was reduced relative to controls, trending toward significance. Little change in Thr 308 phosphorylation was observed. Threonine 308 has been shown to be more closely associated with Akt activity in non-small cell lung cancer samples than serine 473 [34]. With high glucose culture medium, only Ser 473 seemed to be affected by FBXO17 expression, and depletion of FBXO17 did not have significant effects on proliferation (unpublished data). These observations suggest that a delicate balance between serine and threonine phosphorylation can be perturbed by FBXO17 with downstream effects on cell survival and proliferation, and glucose metabolism influences these responses. Phosphorylation of PDK1 as well as mediators further downstream in the PI3K-Akt-mTOR pathway were also activated. Cyclic AMP responsive element binding protein (CREB) activation has been shown to be enhanced by Akt and inhibited by GSK3 $\beta$ [35-37]. Akt-dependent Ser 133 phosphorylation promotes recruitment of CREB binding protein (CBP) to activate gene expression to promote cell survival [37]. Increased FBXO17 levels were associated with increased CREB 


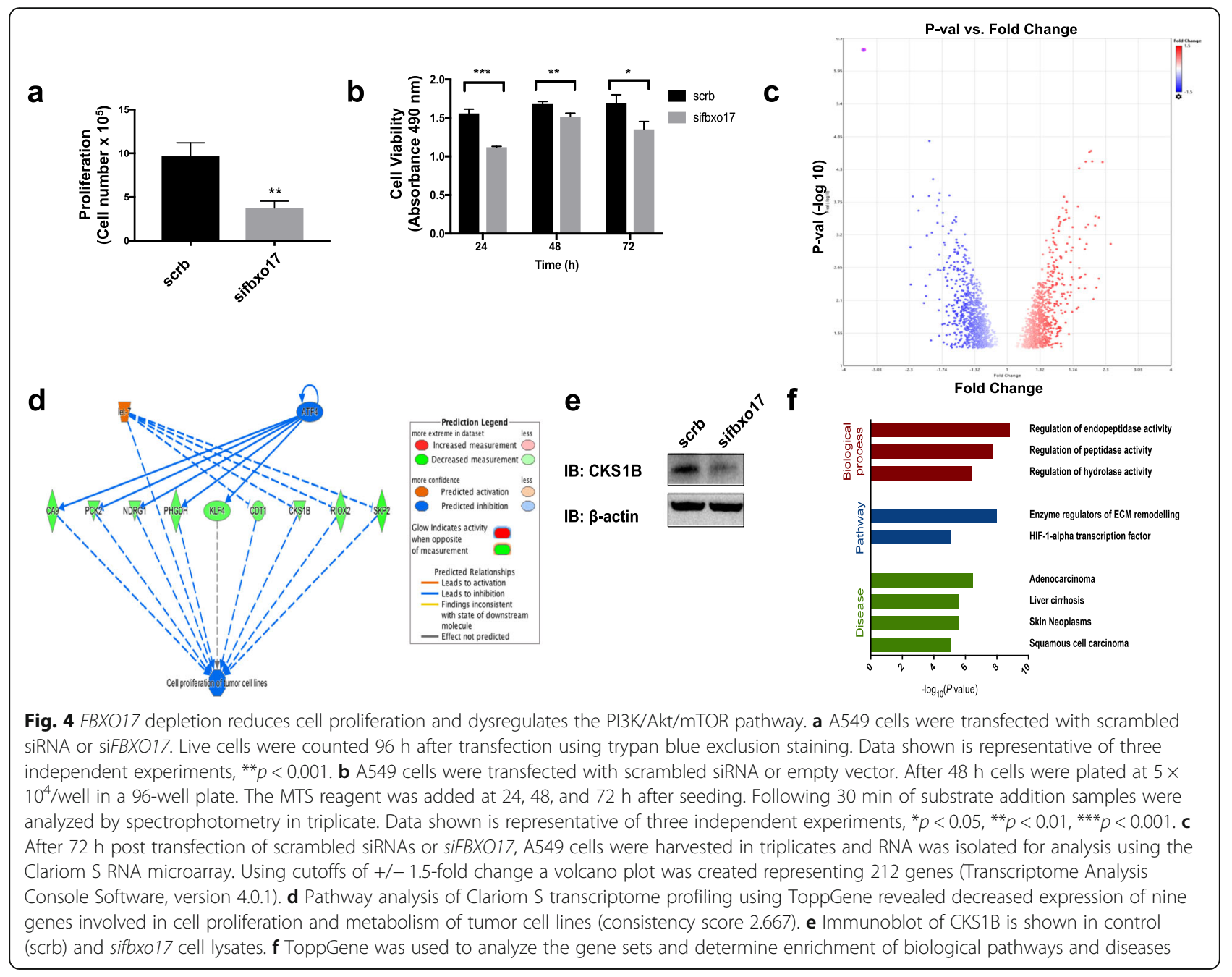

phosphorylation at Ser 133, a site known to enhance CREB activation. Using transcriptome profiling and pathway analysis after FBXO17 knockdown, CKS1B and SKP2 were notable genes that were differentially expressed and important in regulating cell cycle progression through Skp2-mediated degradation of the tumor suppressor p27 ${ }^{\text {Kip }}[38,39]$ Other genes were affected including genes important in metabolism such as PHGDH, RIOX2, and KLF4 that have been shown to have pleotropic roles in chemotherapeutic resistance in different cancers [40-42]. It will be interesting to explore how dysregulation of Akt-dependent pathways by FBXO17 can perturb additional downstream signaling in pathways critical for regulating cell survival and apoptosis.

he magnitude of some of the biological and biochemical processes observed here at times were modest, but can be partly explained by the observation that A549 cells that were used are derived from adenocarcinoma cells with high baseline FBXO17 protein. Thus, overexpression of this F-box protein may have more limited effects biologically given saturation of FBXO17 concentrations in cells that act as a molecular input to pro-survival pathways. ERK1/2 phosphorylation was significantly increased with FBXO17 overexpression and reduced with FBXO17 knockdown, a more pronounced effect while Thr 308 Akt activation is unchanged with FBXO17 knockdown. These data suggest there are alternate mechanisms independent of maximal Akt activation that promote cell proliferation, and future studies will explore the role of the Ras/Raf/ERK kinase pathway in FBXO17-mediated cell proliferation. Prior studies have described ERK-dependent signaling promoting RPS6 phosphorylation [43]. Nevertheless, sustained high cellular concentrations with even modest activation of these cell signaling pathways may translate into a more aggressive cell phenotype with regard to neoplastic behavior. Hence, in future studies the kinetics and magnitude of sustained or conditional FBXO17 expression in a clonal cell line that is devoid of endogenous F-box protein or in vivo system may be of interest.

With the growth of rich databases of genomic data available for lung cancer, molecular details regarding 
mutations, deletions, and amplification of genes can be identified to target therapy. In a recent study by Chen and colleagues, nine subtypes of NSCLC were characterized, and for adenocarcinoma three subtypes showed significant increases in p38 and mTOR pathway activation [44]. Several cancer cell lines have been characterized by having mutations in the mTOR pathway [45]. A high proportion of samples in the original study that formed the basis of The Cancer Genome Atlas showed increased MAPK and mTOR pathway activation, but gene mutations identified could only explain a fraction of these cases [15]. The study illustrates that other pathways leading to activation of mTOR have yet to be identified. Interestingly, a recent study showed that overexpression of a de-ubiquitin enzyme termed ubiquitin C-terminal hydrolase L1 (UCH-L1) promoted Akt activation in MCF-7 breast cancer cells without altering proliferation and enhanced cellular invasion [46]. FBXO17 expression appears to be advantageous for tumor cell survival, and we propose that upregulation of Akt activation by this F-box protein is a potential mechanism for increased mTOR activation in cancer. The results here may be clinically relevant given the interest in personalized, targeted therapies that are currently in use in lung cancer such as use of tyrosine kinase inhibitors for patients with KRAS mutations observed in many NSCLC patients [1, 8, 47, 48].

One limitation of our study is that while we see trends for reduced Akt Ser 473 phosphorylation, this did not reach statistical significance. Akt Thr 308 is consistently unchanged in our experiments with FBXO17 knockdown. CREB is also not significantly reduced with FBXO17 knockdown in line with its role as a downstream target of Akt, suggesting that overall Akt activity remains high, albeit somewhat reduced, with FBXO17 silencing. In our functional assays however, $F B X O 17$ silencing is associated with a significant reduction in cell proliferation, suggesting Akt-independent functional consequences through dysregulated FBXO17 expression. Interestingly we do not observe significant differences in cell cycle progression using serum starvation and BrdU assays (data not shown) suggesting that more robust cellular depletion of $F B X O 17$ may be necessary. We also observed that ERK1/2 was modulated through FBXO17 ectopic expression or depletion, suggesting that other pathways partake in SCF-FBXO17 signaling. In addition, phosphatases PP2A, known to target Thr 308, and PHLPP, which dephosphorylates Ser 473, may be differentially regulated downstream of FBXO17 to explain these results [49]. More complete gene depletion by CRISPR/Cas9 may yield more robust reduction in Akt phosphorylation with FBXO17 gene silencing. High FBXO17 expression, however, drives cell proliferation and may be relevant in the context of lung cancer and aberrant cell proliferation.

\section{Conclusions}

Our studies demonstrate a role for FBXO17 in cell proliferation in lung epithelial cells with some specificity for Ser 473 phosphorylation of Akt. An association was found between FBXO17 expression and lung cancer using the large Cancer Genome Atlas cohort. In addition, downstream targets of Akt including CREB and RPS6 were found to be increased, and ERK1/2 was activated with overexpression of FBXO17, an effect reversed with FBXO17 knockdown, suggesting that these proliferative effects are, in part, Akt-independent. It has also been shown that while RPS6 is phosphorylated by mTOR, Ras/ERK signaling also leads to RPS6 phosphorylation [43, 50]. A subset of lung cancers highly express FBXO17 mRNA transcript and protein, and our data show that FBXO17 promotes robust cell proliferation and cell cycle progression that is potentially relevant in non-small cell lung cancer. These data suggest that while FBXO17 has marked effects on Ser 473 Akt phosphorylation, the F-box protein likely also regulates the Ras/ERK signaling pathway independent of Akt, raising intriguing questions for complementary pathway activation.

It is unclear if the polyubiquitination function of FBXO17 is required for activation of Akt. F-box proteins are capable of recruiting substrates and regulating pathways without proteasomal degradation. For example, FBXO17 itself has been shown to recruit protein phosphatase 2A (PP2A) to interferon regulatory factor 3 (IRF3) to facilitate dephosphorylation of IRF3, thus inhibiting dimerization and translocation to the nucleus [51]. As a result, type I interferon signaling is reduced. Thus, in future studies it is of interest to elucidate if FBXO17 mediates Akt activation through such mechanisms that are not post-translational, or via polyubiquitination and degradation of molecular inputs that inhibit Akt actions. Future studies will also be needed to identify additional substrates of FBXO17 that have potential roles in tumorigenesis and determine if these effects are mediated by polyubiquitination or if FBXO17 serves as a molecular scaffold for other proteins to regulate the PI3K-Akt-mTOR pathway.

\section{Additional files}

Additional file 1: RNA microarray analysis using Transcriptome Analysis Console version 4.0.1. (DOCX $60 \mathrm{~kb}$ )

Additional file 2: Gene ontology enrichment analysis using ToppGene. (DOCX $21 \mathrm{~kb})$

\section{Abbreviations}

4EBP: elF4E binding protein; Akt: Protein kinase B (PKB); BRDU: Bromodeoxyuridine; CKS1B: CDC28 protein kinase regulatory subunit 1B; CREB: Cyclic AMP response element binding protein; DEGs: Differentially expressed genes; DLBCL: Diffuse large B-cell lymphoma; EGFR: Epidermal growth factor receptor; ERK1/2: Extracellular signal-regulated kinase 1/2; FBXO17: F-box only protein 17; GSK33: Glycogen synthase kinase 3 beta; IRF3: Interferon regulatory factor 3; KLF4: Kruppel-like factor 4; MAPK: Mitogen-activated protein kinase; mTOR: Mechanistic target of rapamycin; 
mTORC: Mammalian target of rapamycin complex 1 or 2; MTS: (3-(4,5dimethylthiazol-2-yl)-5-(3-carboxymethoxyphenyl)-2-(4-sulfophenyl)-2H-tetrazolium); NSCLC: Non-small cell lung cancer; PDK1: 3-phosphoinositide-dependent protein kinase-1; PHGDH: Phosphoglycerate dehydrogenase; PHLPP: PH domain and leucine rich repeat protein phosphatase; PI3K: Phosphoinositide 3-kinase; $\mathrm{PIP}_{2}$ : Phosphatidylinositol-4,5-bisphosphate; PIP: Phosphatidylinositol-3,4,5trisphosphate; PP2A: Protein phosphatase 2A; PTEN: Phosphatase and tensin homolog; RIOX2: Ribosomal oxygenase 2; RPS6: Ribosomal protein S6; RTK: Receptor tyrosine kinase; SCF: Skp-Cullin-F-box; Ser: Serine; Thr: Threonine; tSNE: T-distributed stochastic neighbor embedding; VEGF: Vascular endothelial growth factor

\section{Acknowledgements}

The authors thank Brian J. Philips Ph.D. and Ajay Kumar, Ph.D. for providing cancer cell line samples.

\section{Funding}

This work was supported by U.S. National Institute of Health (NIH) Grant R01HL096376, R01HL097376, R01HL098174, R01HL081784, and P01HL114453 and the US Department of Veterans Affairs Office of Research and Development, Biomedical Laboratory Research and Development and a VA Merit Review award to R.K.M; NIH HL131665, HL136294 and American Heart Association Grant number 16GRNT30660001 to Y.Z., NHH HL137248 and Burroughs Wellcome Fund to T.L.S. This project used the University of Pittsburgh HSCRF Genomics Research Core Affymetrix Microarray services.

\section{Availability of data and materials}

The authors will make readily reproducible materials described in the manuscript, including software, databases and all relevant raw data, freely available to scientists.

\section{Authors' contributions}

TS designed and performed experiments, analyzed data and prepared manuscript, IN assisted with experiments and reviewed manuscript, MO analyzed RNA microarray data, $J \mathrm{~L}$ and JZ provided experimental guidance and critique of manuscript, KC analyzed single cell RNA sequencing data, RM and $Y Z$ reviewed manuscript and provided experimental guidance. All authors read and approved the final manuscript.

\section{Ethics approval and consent to participate}

Use of archived human lung tissue was approved by the University of Pittsburgh IRB.

\section{Consent for publication}

N/A

\section{Competing interests}

The authors declare that they have no competing interests.

\section{Publisher's Note}

Springer Nature remains neutral with regard to jurisdictional claims in published maps and institutional affiliations.

\section{Author details \\ ${ }^{1}$ Department of Medicine, the Acute Lung Injury Center of Excellence, University of Pittsburgh, Pittsburgh, PA 15213, USA. ²Department of Cell Biology, Physiology, and Bioengineering, University of Pittsburgh, Pittsburgh, PA 15213, USA. ${ }^{3}$ Medical Specialty Service Line, Veterans Affairs Pittsburgh Healthcare System, Pittsburgh, PA 15240, USA. ${ }^{4}$ Department of Medicine, Pulmonary, Allergy, \& Critical Care Medicine, The University of Pittsburgh, UPMC Montefiore, NW 628, Pittsburgh, PA 15213, USA.}

Received: 6 July 2018 Accepted: 9 October 2018 Published online: 25 October 2018

\section{References}

1. Yip PY. Phosphatidylinositol 3-kinase-AKT-mammalian target of rapamycin (PI3K-Akt-mTOR) signaling pathway in non-small cell lung cancer. Transl Lung Cancer Res. 2015:4:165-76. https://doi.org/10.3978/j.issn.2218-6751. 2015.01.04.
2. Manning BD, Toker A. AKT/PKB signaling: navigating the network. Cell. 2017; 169:381-405. https://doi.org/10.1016/j.cell.2017.04.001.

3. Nakamura N, Ramaswamy S, Vazquez F, Signoretti S, Loda M, et al. Forkhead transcription factors are critical effectors of cell death and cell cycle arrest downstream of PTEN. Mol Cell Biol. 2000;20:8969-82.

4. Leslie NR, Batty $\mathrm{H}$, Maccario H, Davidson L, Downes CP. Understanding PTEN regulation: PIP2, polarity and protein stability. Oncogene. 2008;27: 5464-76. https://doi.org/10.1038/onc.2008.243.

5. Saxton RA, Sabatini DM. mTOR signaling in growth, metabolism, and disease. Cell. 2017;168:960-76. https://doi.org/10.1016/j.cell.2017.02.004.

6. Sarbassov DD, Guertin DA, Ali SM, Sabatini DM. Phosphorylation and regulation of Akt/PKB by the rictor-mTOR complex. Science. 2005;307:1098-101. https://doi.org/10.1126/science.1106148.

7. Menon S, Manning BD. Common corruption of the mTOR signaling network in human tumors. Oncogene. 2008;27(Suppl 2):S43-51. https://doi.org/10. 1038/onc.2009.352.

8. Papadimitrakopoulou V. Development of PI3K/AKT/mTOR pathway inhibitors and their application in personalized therapy for non-small-cell lung cancer. J Thorac Oncol. 2012;7:1315-26. https://doi.org/10.1097/JTO. Ob013e31825493eb.

9. Fumarola C, Bonelli MA, Petronini PG, Alfieri RR. Targeting PI3K/AKT/mTOR pathway in non small cell lung cancer. Biochem Pharmacol. 2014;90:197207. https://doi.org/10.1016/j.bcp.2014.05.011.

10. Song MS, Salmena L, Pandolfi PP. The functions and regulation of the PTEN tumour suppressor. Nat Rev Mol Cell Biol. 2012;13:283-96. https://doi.org/10. 1038/nrm3330.

11. Liu Y, Mallampalli RK. Small molecule therapeutics targeting F-box proteins in cancer. Semin Cancer Biol. 2016;36:105-19. https://doi.org/10.1016/j. semcancer.2015.09.014.

12. Weathington NM, Mallampalli RK. New insights on the function of SCF ubiquitin E3 ligases in the lung. Cell Signal. 2013;25:1792-8. https://doi.org/ 10.1016/j.cellsig.2013.05.003.

13. Sato T, Nakashima A, Guo L, Coffman K, Tamanoi F. Single amino-acid changes that confer constitutive activation of mTOR are discovered in human cancer. Oncogene. 2010;29:2746-52. https://doi.org/10.1038/onc.2010.28.

14. McDonald JM, Pelloski CE, Ledoux A, Sun M, Raso G, et al. Elevated phospho-S6 expression is associated with metastasis in adenocarcinoma of the lung. Clin Cancer Res. 2008;14:7832-7. https://doi.org/10.1158/10780432.CCR-08-0565.

15. Cancer Genome Atlas Research Network. Comprehensive molecular profiling of lung adenocarcinoma. Nature. 2014;511:543-50. https://doi.org/ 10.1038/nature13385.

16. Dibble CC, Cantley LC. Regulation of mTORC1 by PI3K signaling. Trends Cell Biol. 2015;25:545-55. https://doi.org/10.1016/j.tcb.2015.06.002.

17. Díaz VM, de Herreros AG. F-box proteins: keeping the epithelial-tomesenchymal transition (EMT) in check. Semin Cancer Biol. 2016:36:71-9. https://doi.org/10.1016/j.semcancer.2015.10.003.

18. Zhang Y, Nicholatos J, Dreier JR, Ricoult SJH, Widenmaier SB, et al. Coordinated regulation of protein synthesis and degradation by MTORC1. Nature. 2014;513:440-3. https://doi.org/10.1038/nature13492.

19. Weathington NM, Mallampalli RK. Emerging therapies targeting the ubiquitin proteasome system in cancer. J Clin Invest. 2014;124:6-12. https://doi.org/10.1172/JCl71602.

20. Jin J, Cardozo T, Lovering RC, Elledge SJ, Pagano M, et al. Systematic analysis and nomenclature of mammalian F-box proteins. Genes Dev. 2004; 18:2573-80. https://doi.org/10.1101/gad.1255304.

21. Ravid T, Hochstrasser M. Diversity of degradation signals in the ubiquitinproteasome system. Nat Rev Mol Cell Biol. 2008;9:679-90. https://doi.org/10. 1038/nrm2468.

22. Guharoy M, Bhowmick P, Sallam M, Tompa P. Tripartite degrons confer diversity and specificity on regulated protein degradation in the ubiquitinproteasome system. Nat Commun. 2016;7:10239. https://doi.org/10.1038/ ncomms10239.

23. Minella AC, Clurman BE. Mechanisms of tumor suppression by the SCF(Fbw7). Cell Cycle. 2005;4:1356-9. https://doi.org/10.4161/cc.4.10.2058.

24. Akhoondi S, Sun D, von der Lehr N, Apostolidou S, Klotz K, et al. FBXW7/ hCDC4 is a general tumor suppressor in human cancer. Cancer Res. 2007;67: 9006-12. https://doi.org/10.1158/0008-5472.CAN-07-1320.

25. Duan S, Cermak L, Pagan JK, Rossi M, Martinengo C, et al. FBXO11 targets BCL6 for degradation and is inactivated in diffuse large B-cell lymphomas. Nature. 2012;481:90-3. https://doi.org/10.1038/nature10688. 
26. Suber T, Wei J, Jacko AM, Nikolli I, Zhao Y, et al. SCFFBXO17 E3 ligase modulates inflammation by regulating proteasomal degradation of glycogen synthase kinase-3 $\beta$ in lung epithelia. J Biol Chem. 2017;292:7452-61. https://doi.org/10.1074/jbc.M116.771667.

27. Chen J, Bardes EE, Aronow BJ, Jegga AG. ToppGene suite for gene list enrichment analysis and candidate gene prioritization. Nucleic Acids Res. 2009;37:W305-11. https://doi.org/10.1093/nar/gkp427.

28. Schneider CA, Rasband WS, Eliceiri KW. NIH image to ImageJ: 25 years of image analysis. Nat Methods. 2012;9:671-5. https://doi.org/10.1038/nmeth.2089.

29. Gao J, Aksoy BA, Dogrusoz U, Dresdner G, Gross B, et al. Integrative analysis of complex cancer genomics and clinical profiles using the cBioPortal. Sci Signal. 2013;6:pl1. https://doi.org/10.1126/scisignal.2004088.

30. Cerami E, Gao J, Dogrusoz U, Gross BE, Sumer SO, et al. The cBio cancer genomics portal: an open platform for exploring multidimensional cancer genomics data. Cancer Discov. 2012;2:401-4. https://doi.org/10.1158/21598290.CD-12-0095.

31. Cohen P, Frame S. The renaissance of GSK3. Nat Rev Mol Cell Biol. 2001;2: 769-76. https://doi.org/10.1038/35096075.

32. Alessi DR, Andjelkovic M, Caudwell B, Cron P, Morrice N, et al. Mechanism of activation of protein kinase B by insulin and IGF-1. EMBO J. 1996;15:6541-51.

33. Guo H, Gao M, Lu Y, Liang J, Lorenzi PL, et al. Coordinate phosphorylation of multiple residues on single AKT1 and AKT2 molecules. Oncogene. 2014:33:3463-72. https://doi.org/10.1038/onc.2013.301.

34. Vincent EE, Elder DJE, Thomas EC, Phillips L, Morgan C, et al. Akt phosphorylation on Thr308 but not on Ser473 correlates with Akt protein kinase activity in human non-small cell lung cancer. Br J Cancer. 2011;104: 1755-61. https://doi.org/10.1038/bjc.2011.132.

35. Tullai JW, Chen J, Schaffer ME, Kamenetsky E, Kasif S, et al. Glycogen synthase kinase-3 represses cyclic AMP response element-binding protein (CREB)-targeted immediate early genes in quiescent cells. J Biol Chem. 2007;282:9482-91. https://doi.org/10.1074/jbc.M700067200.

36. Daniel P, Filiz G, Brown DV, Hollande F, Gonzales M, et al. Selective CREBdependent cyclin expression mediated by the PI3K and MAPK pathways supports glioma cell proliferation. Oncogenesis. 2014;3:e108. https://doi.org/ 10.1038/oncsis.2014.21

37. Du K, Montminy M. CREB is a regulatory target for the protein kinase Akt/ PKB. J Biol Chem. 1998;273:32377-9.

38. Hao B, Zheng N, Schulman BA, Wu G, Miller JJ, et al. Structural basis of the Cks1-dependent recognition of p27(Kip1) by the SCF(Skp2) ubiquitin ligase. Mol Cell. 2005;20:9-19. https://doi.org/10.1016/j.molcel.2005.09.003.

39. Krishnan A, Nair SA, Pillai MR. Loss of cks1 homeostasis deregulates cell division cycle. J Cell Mol Med. 2010;14:154-64. https://doi.org/10.1111/j. 1582-4934.2009.00698.x.

40. Lee SI, Kim DK, Seo EJ, Choi EJ, Kwon YW, et al. Role of Krüppel-like factor 4 in the maintenance of Chemoresistance of anaplastic thyroid Cancer. Thyroid. 2017;27:1424-32. https://doi.org/10.1089/thy.2016.0414.

41. Feng W, Xie Q, Liu S, Ji Y, Li C, et al. Krüppel-like factor 4 promotes c-met amplification-mediated gefitinib resistance in non-small-cell lung cancer. Cancer Sci. 2018;109:1775-86. https://doi.org/10.1111/cas.13601.

42. Wang B, Shen A, Ouyang X, Zhao G, Du Z, et al. KLF4 expression enhances the efficacy of chemotherapy drugs in ovarian cancer cells. Biochem Biophys Res Commun. 2017;484:486-92. https://doi.org/10.1016/j.bbrc.2017.01.062.

43. Roux PP, Shahbazian D, Vu H, Holz MK, Cohen MS, et al. RAS/ERK signaling promotes site-specific ribosomal protein S6 phosphorylation via RSK and stimulates cap-dependent translation. J Biol Chem. 2007;282:14056-64. https://doi.org/10.1074/jbc.M700906200.

44. Chen F, Zhang Y, Parra E, Rodriguez J, Behrens C, et al. Multiplatform-based molecular subtypes of non-small-cell lung cancer. Oncogene. 2017;36:138493. https://doi.org/10.1038/onc.2016.303.

45. Grabiner BC, Nardi V, Birsoy K, Possemato R, Shen K, et al. A diverse array of cancer-associated MTOR mutations are hyperactivating and can predict rapamycin sensitivity. Cancer Discov. 2014;4:554-63. https://doi.org/10.1158/ 2159-8290.CD-13-0929.

46. Luo Y, He J, Yang C, Orange M, Ren X, et al. UCH-L1 promotes invasion of breast cancer cells through activating Akt signaling pathway. J Cell Biochem. 2018;119:691-700. https://doi.org/10.1002/jcb.26232.

47. Jiang Z-B, Huang J, Xie C, Li X, Liu L, et al. Combined use of PI3K and MEK inhibitors synergistically inhibits lung cancer with EGFR and KRAS mutations. Oncol Rep. 2016;36:365-75. https://doi.org/10.3892/or.2016.4770.
48. Zer A, Leighl N. Promising targets and current clinical trials in metastatic non-squamous NSCLC. Front Oncol. 2014;4:329. https://doi.org/10.3389/fonc. 2014.00329

49. Bayascas JR, Alessi DR. Regulation of Akt/PKB Ser473 phosphorylation. Mol Cell. 2005;18:143-5. https://doi.org/10.1016/j.molcel.2005.03.020.

50. Mendoza MC, Er EE, Blenis J. The Ras-ERK and PI3K-mTOR pathways: crosstalk and compensation. Trends Biochem Sci. 2011;36:320-8. https://doi.org/ 10.1016/j.tibs.2011.03.006.

51. Peng D, Wang Z, Huang A, Zhao Y, Qin FX-F. A novel function of F-box protein $\mathrm{FBXO17}$ in negative regulation of type I IFN signaling by recruiting PP2A for IFN regulatory factor 3 deactivation. J Immunol. 2017;198:808-19. https://doi.org/10.4049/jimmunol.1601009.
Ready to submit your research? Choose BMC and benefit from:

- fast, convenient online submission

- thorough peer review by experienced researchers in your field

- rapid publication on acceptance

- support for research data, including large and complex data types

- gold Open Access which fosters wider collaboration and increased citations

- maximum visibility for your research: over $100 \mathrm{M}$ website views per year

At BMC, research is always in progress.

Learn more biomedcentral.com/submissions 